\title{
MEK Inhibitor GDC-0623
}

National Cancer Institute

\section{Source}

National Cancer Institute. MEK Inhibitor GDC-0623. NCI Thesaurus. Code C95738.

An orally active, selective MEK inhibitor with potential antineoplastic activity. MEK inhibitor GDC-0623 specifically inhibits mitogen-activated protein kinase kinase (MEK or MAP/ERK kinase), resulting in inhibition of growth factor-mediated cell signaling and tumor cell proliferation. MEK is a key component of the RAS/RAF/MEK/ERK signaling pathway that regulates cell growth; constitutive activation of this pathway has been implicated in many cancers. 